\title{
Physiopathologie
}

\section{Progression des allergies et régression des maladies infectieuses}

Les manifestations «allergiques » des voies respiratoires sont de plus en plus fréquentes. Lorsqu'elles concernent les voies respiratoires inférieures, elles provoquent la constriction et l'obstruction des bronches, ce qui entraîne des crises d'asthme. Les bronches des sujets asthmatiques se contractent en réponse à des doses très faibles de bronchoconstricteurs (comme l'histamine) ou de substances toxiques (comme le tabac). Cela peut bloquer le passage de l'air surtout si les bronches sont déjà partiellement obstruées par du mucus sécrété en excès sous l'action combinée des mastocytes, des basophiles et des éosinophiles [1].

L'atopie caractérise un état allergique en réponse à des antigènes de l'environnement, les allergènes. Les manifestations cliniques de l'atopie sont nombreuses et regroupent aussi bien le rhume des foins que l'eczéma et l'asthme. L'atopie peut être reconnue par la présence dans le sérum d'IgE spécifiques de l'allergène ou par des réactions d'hypersensibilité immédiate aux allergènes à la suite d'un test cutané intradermique. Comme les symptômes, les allergènes sont très variés. Le plus souvent il s'agit de poussières organiques provenant d'acariens ou de pollens. Parmi les facteurs intrinsèques favorisant l'atopie, la population $\mathrm{T}_{\mathrm{H}} 2$ des lymphocytes $\mathrm{T}$ CD4 est essentielle. En effet, lorsqu'ils sont activés, les lymphocytes $\mathrm{T}_{\mathrm{H}} 2$ sécrètent surtout de l'interleukine 4 (IL-4), de l'IL-10 et de l'IL-13 et peuvent stimuler la production d'IgE et le développement de l'hypersensibilité immédiate.

La sévérité et la prévalence des maladies atopiques se sont fortement accrues au cours des dernières années dans les pays industrialisés. La pollution de l'air et les modifications du mode de vie (exposition croissante aux poussières organiques, habitudes alimentaires, etc.) ont été largement incriminées sans que l'on puisse réellement déterminer la cause de cette augmentation. Les fréquences de l'asthme dans les pays «propres» du Nord de l'Europe et dans les pays pollués d'Europe de l'Est indiquent même que la pollution de l'air serait plus un facteur aggravant de l'atopie qu'un facteur causal direct.

L'augmentation de la prévalence de l'asthme pourrait être due à la disparition de facteurs de résistance plutôt qu'à l'action favorisante de facteurs toxiques. Par exemple, la prévalence de l'asthme dans les familles nombreuses est plus faible chez les enfants les plus jeunes alors qu'ils sont plus sujets aux infections, notamment aux infections respiratoires, que leurs ainés. De même, les infections sont plus fréquentes dans les populations de l'ancien bloc de l'Est que dans les pays d'Europe de l'Ouest où l'asthme est le plus courant. Il est donc possible que certaines infections soient un facteur de protection contre l'asthme. Ainsi, le déclin des maladies infectieuses dans les pays industrialisés pourrait-il être associé à la recrudescence de l'atopie. C'est en tout cas ce que suggère une étude effectuée au Japon et publiée dans la revue Science [2].

Les lymphocytes $\mathrm{T}_{\mathrm{H}} 1$ sécrètent de l'IL2, de l'interféron $\gamma$, du TNF $\alpha$ (tumor necrosis factor $\alpha$ ) et inhibent les fonctions de la population lymphocytaire $\mathrm{T}_{\mathrm{H}} 2$. Grâce à leur potentiel inhibiteur des fonctions $\mathrm{T}_{\mathrm{H}} 2$, les maladies infectieuses, comme la tuberculose, provoquant une forte réponse immunitaire de type $T_{H} 1$ seraient autant de facteurs de protection contre l'atopie. La disparition de ces maladies conduirait à une utilisation moins bien contrôlée de la réponse immunitaire de type $\mathrm{T}_{\mathrm{H}} 2$, à la production inappropriée d'IgE et à l'hypersensibilité immédiate.

Un bon indicateur de la réponse de type $\mathrm{T}_{\mathrm{H}} 1$ est le développement de l'hypersensibilité retardée en réaction à l'injection intradermique de tuberculine. Ce test a été utilisé par Shirakawa et al. pour montrer que les individus capables de développer une bonne réponse immunitaire de type $\mathrm{T}_{\mathrm{H}} 1$ sont moins sujets que les autres aux désordres atopiques en général et à l'asthme en particulier [2]. Cette étude a été menée au Japon dans la province de Wakayama où un programme de vaccination par le BCG a été particulièrement bien suivi. L'étude porte sur 867 enfants de 1213 ans ayant un mode de vie semblable et un passé médical bien documenté.

La positivité de la réaction à la tuberculine est liée à l'âge des enfants: $3 \%$ à 3 mois, $33,2 \%$ à 6 mois et $58 \%$ à 12 ans. Les symptômes courants de l'atopie sont 3 fois plus fréquents chez les enfants ne répondant pas à la tuberculine. En revanche, la rémission des symptômes, entre 7 et 12 ans, est 6 à 9 fois plus fréquentes chez les sujets ayant un test positif à la tuberculine. Les taux d'IgE totales et spécifiques d'allergènes ainsi que la concentration de cytokines de type $\mathrm{T}_{\mathrm{H}} 2$ sont plus faibles chez les sujets positifs pour le test à la tuberculine. A l'inverse, la concentration des cytokines de type $\mathrm{T}_{\mathrm{H}} 1$ est plus élevée chez ces individus. 
Ces résultats suggèrent qu'une bonne utilisation de la composante $\mathrm{T}_{\mathrm{H}} 1 \mathrm{du}$ système immunitaire réduit le risque de développement de réactions allergiques. Les auteurs proposent que certaines infections protègent de l'atopie en fortifiant la composante $\mathrm{T}_{\mathrm{H}} 1 \mathrm{du}$ système immunitaire. La tuberculose pourrait être l'une de ces infections. Elle induit une forte réponse de type $T_{\mathrm{H}} 1$ et est en permanente régression dans la région de Watayama où les maladies atopiques sont de plus en plus fréquentes.
Manipuler le système immunitaire de telle sorte que l'équilibre $\mathrm{T}_{\mathrm{H}} 1 / \mathrm{T}_{\mathrm{H}} 2$ penche en faveur de la composante $\mathrm{T}_{\mathrm{H}} 1$ est un moyen imaginable pour lutter contre les allergies. Il faut admettre, toutefois, qu'un tel déséquilibre ne doit pas être trop instable pour être durable. Cela n'est pas prouvé et il semble au contraire qu'un déséquilibre $\mathrm{T}_{\mathrm{H}} 1 / \mathrm{T}_{\mathrm{H}} 2$ ne puisse se maintenir qu'en présence d'un agent infectieux. Quoi qu'il en soit, et même si elle n'est que circonstancielle, une relation est établie entre la régression des maladies infectieuses et la recrudescence des maladies atopiques.

V.L.

1. Benvéniste J, Marsac J. Asthme, hyper-réactivité bronchique et inflammation. Med Sci 1987; 3: 506-7.

2. Shirakawa T, Enomoto T, Shimazu S, Hopkin J.M. The inverse association between tuberculin responses and atopic disorder. Science 1997; 275 : $77-9$.

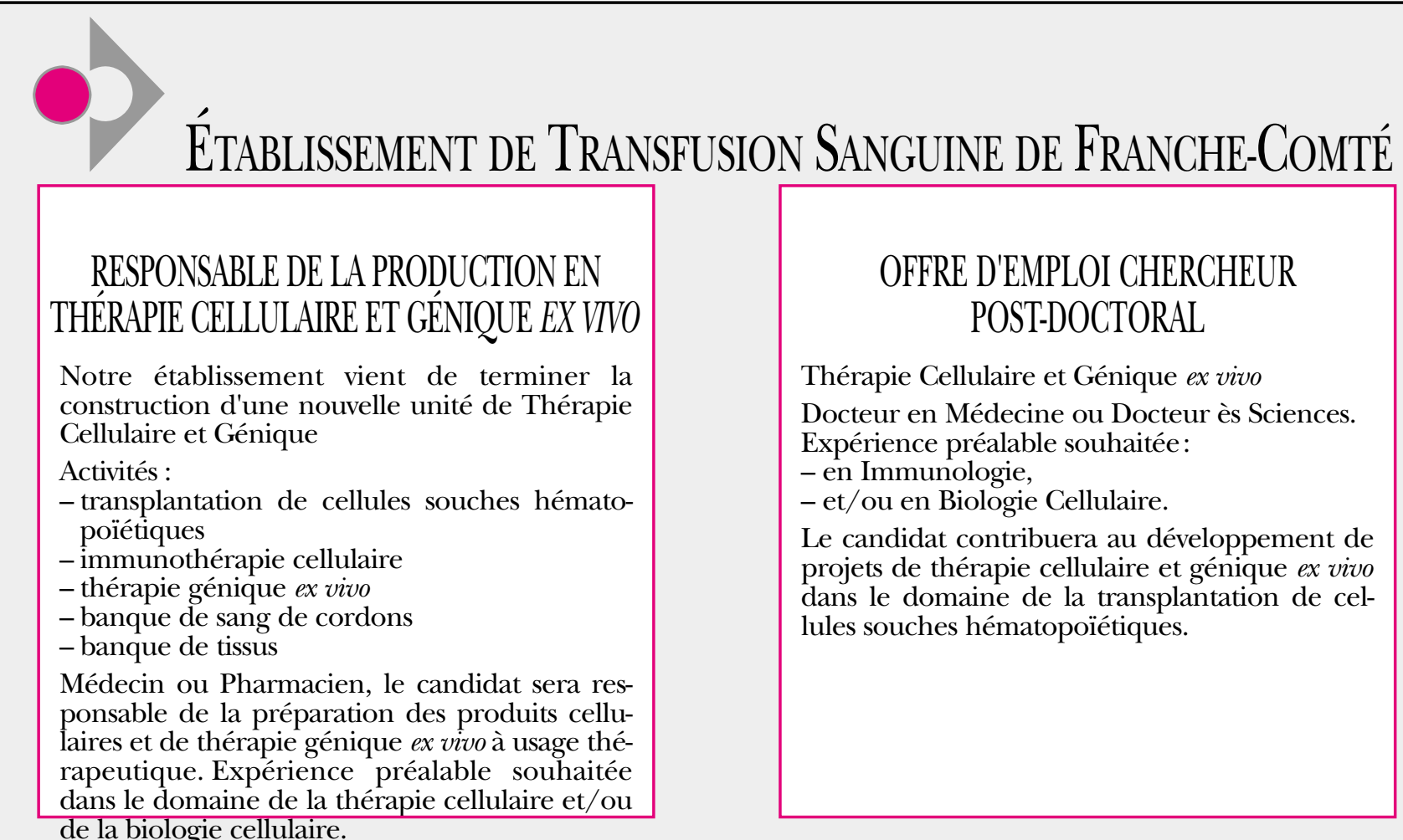

Les candidats devront envoyer un CV et les coordonnées de deux références au Docteur Pierre TIBERGHIEN - Etablissement de Transfusion Sanguine de Franche-Comté 1. boulevard Fleming - 25000 Besancon - France - Tél. :03 81 61 56 15 - Fax:03 816156 17 E-mail : pierre.tiberghein@univ-fcomte.fr 